\title{
VORTEX FILAMENT EQUATION IN A RIEMANNIAN MANIFOLD
}

\author{
NORIHITO KOISO \\ (Received May 28, 2001, revised July 23, 2002)
}

\begin{abstract}
We define a riemannian version of the vortex filament equation. Using perturbation to a parabolic equation, we prove the short time unique existence of a solution for any initial closed curve.
\end{abstract}

1. Introduction and preliminaries. The vortex filament equation is an equation of a curve $\gamma(x, t)$ in the three-dimensional euclidean space:

$$
\gamma_{t}=\gamma_{x} \times \gamma_{x x}, \quad\left|\gamma_{x}\right| \equiv 1
$$

where $x$ is the exterior product. Hasimoto $[\mathrm{H}]$ showed that this equation can be transformed to a standard nonlinear Schrödinger equation. However, his transformation was not mathematically well-defined.

The existence of a solution of (V) was first proved by Nishiyama and Tani [NT] using a perturbation to a fourth order parabolic equation. The present author gave another proof using a perturbation to a second order parabolic equation, and justified mathematically Hasimoto's transformation $[\mathrm{K}]$.

For a solution $\gamma(x, t)$ of $(\mathrm{V}), \xi:=\gamma_{x}$ satisfies $\xi_{t}=\xi \times \xi_{x x}$. Moreover, the norm $|\xi|$ is preserved along time. Therefore, the equation of $\xi$ becomes an equation in the standard sphere $S^{2}$ in the euclidean three-space. This is a key point of the proofs in both [NT] and [K]. We can perturb the equation of $\xi$ to a parabolic equation in $S^{2}$.

In this paper, we consider the vortex filament equation in a general oriented threedimensional Riemannian manifold $(M, g)$ :

$$
\gamma_{t}=\gamma_{x} \times \nabla_{x} \gamma_{x}, \quad\left|\gamma_{x}\right| \equiv 1
$$

where $\nabla$ is the covariant differentiation. When $(M, g)$ is homogeneous, we can generalize the above technique, and obtain the existence of a short time solution [K].

Our main interest is the stability of Equation (V) under the most natural generalization from a point of view of Riemannian geometry.

In the euclidean space, Hasimoto's transformation reduces Equation (V) of three unknown functions to an equation of two unknown functions. However, in a general Riemannian manifold, such a transformation converts Equation (V) to an equation of five unknown functions, because the equation contains position variables.

2000 Mathematics Subject Classification. Primary 53C44; Secondary 35Q35, 35B25.

Key words and phrases. Vortex filament, Riemannian manifold, singular perturbation. 
Therefore, we have to take more direct approach. We perturb the equation of $\gamma$ itself to a parabolic equation:

$$
\gamma_{t}=\gamma_{x} \times \nabla_{x} \gamma_{x}+\varepsilon \nabla_{x} \gamma_{x} .
$$

Nishiyama $[\mathrm{N}]$ took this approach in a different setting. He proved the existence of a solution, but did not show its uniqueness. The difficulty is caused by the variation of the norm $\left|\gamma_{x}\right|$ along time. We will overcome this difficulty by estimating $\gamma$ and $w:=\left|\gamma_{x}\right|^{2}$ simultaneously, and prove

THEOREM 3.1. The equation (VM) has a unique short time solution for any $C^{\infty}$ closed initial curve $\gamma_{0}(x)$ with $\left|\nabla_{x} \gamma_{0}\right| \equiv 1$.

We here summarize our notation. We denote by $|*|$ the pointwise norm, by $\nabla$ the covariant differentiation, by $R$ the curvature tensor, and by $\times$ the exterior product on each tangent space $T_{p} M$, respectively. Partial derivation is denoted by subscript or $\partial_{x}, \partial_{t}$ :

$$
\eta_{u}=\partial_{u} \eta:=\partial_{u} \eta^{i} \frac{\partial}{\partial x^{i}}=\frac{\partial \eta^{i}}{\partial u} \frac{\partial}{\partial x^{i}} .
$$

The manifold $M$, its structure and all functions on $M$ are supposed to be of class $C^{\infty}$. We may assume that the curvature and its derivatives are bounded on $M$, because we only consider the short time existence.

For convenience, we recall relevant basic facts from Riemannian geometry. For a map $\eta=\eta(u, v): \boldsymbol{R}^{2} \rightarrow M, \eta_{u}$ is a vector field along the map $\eta$. The covariant derivative $\nabla_{u} X$ of a vector field $X$ along $\eta$ for $u$-direction is given by

$$
\nabla_{u} X=\left(\nabla_{u} X\right)^{i} \frac{\partial}{\partial x^{i}}=\left\{\partial_{u} X^{i}+\Gamma(\eta)_{j}{ }^{i} k \cdot \partial_{u} \eta^{j} \cdot X^{k}\right\} \frac{\partial}{\partial x^{i}},
$$

where $\Gamma_{j}{ }_{k}{ }_{k}$ are Christoffel's symbols. We see $\nabla_{u} \eta_{v}=\nabla_{v} \eta_{u}$ by definition, but higher covariant differentiations do not commute: $\nabla_{v} \nabla_{u} X-\nabla_{u} \nabla_{v} X=R\left(\eta_{v}, \eta_{u}\right) X$. The curvature tensor $R$ has many symmetries, but we will not use them. The Riemannian metric $g$ and the exterior product $\times$ are parallel with respect to the covariant differentiation: $\partial_{u}\{g(X, Y)\}=$ $g\left(\nabla_{u} X, Y\right)+g\left(X, \nabla_{u} Y\right), \nabla_{u}(X \times Y)=\left(\nabla_{u} X\right) \times Y+X \times\left(\nabla_{u} Y\right)$.

We may assume, by rescaling, that the initial length of the curve is 1 . Therefore, we may consider $\gamma$ as a map from $(\boldsymbol{R} / \boldsymbol{Z}) \times \boldsymbol{R}_{\geq 0}$ to $M$.

We will take function norms only for $x$-direction. More precisely, we define the $L_{2}$ inner product $\langle *, *\rangle$ and the $L_{2}$ norm $\|*\|$ as follows.

$$
\langle\alpha, \beta\rangle:=\int_{0}^{1} g(\alpha, \beta) d x, \quad\|\alpha\|^{2}:=\langle\alpha, \alpha\rangle, \quad\|\alpha\|_{n}^{2}=\sum_{i=0}^{n}\left\|\nabla_{x}^{i} \alpha\right\|^{2} .
$$

Also, $\|\alpha\|_{C^{n}}$ measures only $x$-derivatives and is a function in $t$. By integration by parts, we have $\left\langle\nabla_{x} X, Y\right\rangle=-\left\langle X, \nabla_{x} Y\right\rangle$.

2. Existence. In this section we consider Problem (P) with a closed initial curve $\gamma_{0}(x)$ such that $\left|\gamma_{0 x}\right| \equiv 1$. We assume that $0<\varepsilon \leq 1$. Then (P) becomes parabolic, and a short time 
solution $\gamma(x, t)$ exists for each $\varepsilon$ (see, e.g., [E, Theorem 6.3]. We apply it to periodic functions on $\boldsymbol{R}$ ). In the following, we denote by $C, C_{i}, K$ and $T$ positive constants independent of $\varepsilon$.

LEMMA 2.1. $w_{t}=\varepsilon\left(w_{x x}-2\left|\nabla_{x} \gamma_{x}\right|^{2}\right)$.

Proof. It follows from a simple calculation that

$$
\begin{aligned}
w_{t} & =2 g\left(\gamma_{x}, \nabla_{t} \gamma_{x}\right)=2 g\left(\gamma_{x}, \nabla_{x} \gamma_{t}\right)=2 g\left(\gamma_{x}, \nabla_{x} \gamma_{x} \times \nabla_{x} \gamma_{x}+\gamma_{x} \times \nabla_{x}^{2} \gamma_{x}+\varepsilon \nabla_{x}^{2} \gamma_{x}\right) \\
& =2 \varepsilon\left\{\partial_{x}\left(g\left(\gamma_{x}, \nabla_{x} \gamma_{x}\right)\right)-\left|\nabla_{x} \gamma_{x}\right|^{2}\right\}=\varepsilon\left(w_{x x}-2\left|\nabla_{x} \gamma_{x}\right|^{2}\right) .
\end{aligned}
$$

LEMmA 2.2. It holds that $\max w \leq 1$ and $\left\|\nabla_{x} \gamma_{x}\right\|,\left\|w_{x}\right\| \leq C$.

Proof. By the maximum principle, Lemma 2.1 implies that $\max w \leq 1$, i.e.,

$$
\begin{aligned}
\limsup _{h \downarrow 0} & \frac{1}{h}\{\max w(*, t)-\max w(*, t-h)\} \leq \limsup _{h \downarrow 0} \frac{1}{h}\{w(x, t)-w(x, t-h)\} \\
= & w_{t}(x, t) \leq \varepsilon w_{x x}(x, t) \leq 0,
\end{aligned}
$$

where $x$ is the maximum point of $w$ at $t$. For $\left\|\nabla_{x} \gamma_{x}\right\|$, using integration by parts, we have

$$
\begin{aligned}
\frac{d}{d t}\left\|\nabla_{x} \gamma_{x}\right\|^{2} & =2\left\langle\nabla_{x} \gamma_{x}, \nabla_{t} \nabla_{x} \gamma_{x}\right\rangle=2\left\langle\nabla_{x} \gamma_{x}, R\left(\gamma_{t}, \gamma_{x}\right) \gamma_{x}+\nabla_{x}^{2} \gamma_{t}\right\rangle \\
& =2\left\langle\nabla_{x} \gamma_{x}, R\left(\gamma_{x} \times \nabla_{x} \gamma_{x}+\varepsilon \nabla_{x} \gamma_{x}, \gamma_{x}\right) \gamma_{x}\right\rangle-2\left\langle\nabla_{x}^{2} \gamma_{x}, \gamma_{x} \times \nabla_{x}^{2} \gamma_{x}+\varepsilon \nabla_{x}^{2} \gamma_{x}\right\rangle \\
& \leq C_{1}\left\|\nabla_{x} \gamma_{x}\right\|^{2}-2 \varepsilon\left\|\nabla_{x}^{2} \gamma_{x}\right\|^{2},
\end{aligned}
$$

which means that $\left\|\nabla_{x} \gamma_{x}\right\|$ increases at most exponentially. Consequently, we see that $\left\|w_{x}\right\|=$ $\left\|2 g\left(\gamma_{x}, \nabla_{x} \gamma_{x}\right)\right\| \leq 2\left\|\nabla_{x} \gamma_{x}\right\| \leq C_{2}$.

LEMma 2.3. There exists a positive constant $T$ such that $w \geq 1 / 2$ holds for any solution $\gamma(x, t)$ defined on a subinterval $\left[0, T^{\prime}\right)$ of $[0, T)$.

Proof. By Lemma 2.2, we have $\left\|\nabla_{x} \gamma_{x}\right\|,\left\|w_{x}\right\| \leq C_{1}$. Hence, from

$$
\begin{aligned}
\frac{d}{d t}\|w\|^{2} & =2\left\langle w, w_{t}\right\rangle=2 \varepsilon\left\langle w, w_{x x}-2\left|\nabla_{x} \gamma_{x}\right|^{2}\right\rangle=-2 \varepsilon\left\|w_{x}\right\|^{2}-4 \varepsilon\left\langle w,\left|\nabla_{x} \gamma_{x}\right|^{2}\right\rangle \\
& \geq-C_{2}-4\left\|\nabla_{x} \gamma_{x}\right\|^{2} \geq-C_{3},
\end{aligned}
$$

we see that $\|w\|^{2} \geq 1-C_{3} T$ holds on $0 \leq t<T$, and that

$$
\|1-w\|^{2} \leq\|1-w\|^{2}+\|w\|^{2}-1+C_{3} T \leq 2\langle w, w-1\rangle+C_{3} T \leq C_{3} T .
$$

Therefore, by the Sobolev imbedding theorem,

$$
\max (1-w)^{2} \leq\|1-w\|\left(\|1-w\|+\left\|w_{x}\right\|\right) \leq C_{4} \sqrt{T}\left(\sqrt{T}+C_{5}\right),
$$

and the result holds for a small $T$. 
LEMMA 2.4. Let $\gamma(x, t)$ be as above. There exists a positive constant $C$ such that

$$
\begin{aligned}
& \varepsilon^{-1} \frac{d}{d t}\left\|w_{x}\right\|^{2} \leq-\left\|w_{x x}\right\|^{2}+C\left(1+\left\|\nabla_{x}^{2} \gamma_{x}\right\|\right), \\
& \varepsilon^{-1} \frac{d}{d t}\left\|w_{x x}\right\|^{2} \leq-\left\|w_{x x x}\right\|^{2}+C\left(1+\left\|\nabla_{x}^{2} \gamma_{x}\right\|^{3}\right) .
\end{aligned}
$$

PROOF.

$$
\begin{aligned}
\frac{d}{d t}\left\|w_{x}\right\|^{2} & =2\left\langle w_{x}, w_{t x}\right\rangle=-2 \varepsilon\left\langle w_{x x}, w_{x x}-2\left|\nabla_{x} \gamma_{x}\right|^{2}\right\rangle \\
& =-2 \varepsilon\left\|w_{x x}\right\|^{2}+4 \varepsilon\left\langle w_{x x},\left|\nabla_{x} \gamma_{x}\right|^{2}\right\rangle \leq-\varepsilon\left\|w_{x x}\right\|^{2}+C_{1} \varepsilon\left\|\nabla_{x} \gamma_{x}\right\|^{2}\left\|\nabla_{x} \gamma_{x}\right\|_{C^{0}}^{2}, \\
\frac{d}{d t}\left\|w_{x x}\right\|^{2} & =2\left\langle w_{x x}, w_{t x x}\right\rangle=-2 \varepsilon\left\langle w_{x x x}, w_{x x x}-4 g\left(\nabla_{x} \gamma_{x}, \nabla_{x}^{2} \gamma_{x}\right)\right\rangle \\
& =-2 \varepsilon\left\|w_{x x x}\right\|^{2}+8 \varepsilon\left\langle w_{x x x}, g\left(\nabla_{x} \gamma_{x}, \nabla_{x}^{2} \gamma_{x}\right)\right\rangle \\
& \leq-\varepsilon\left\|w_{x x x}\right\|^{2}+C_{2} \varepsilon\left\|\nabla_{x} \gamma_{x}\right\|_{C^{0}}^{2}\left\|\nabla_{x}^{2} \gamma_{x}\right\|^{2} .
\end{aligned}
$$

PROPOSITION 2.5. There exist positive constants $T$ and $C$ such that $\left\|\nabla_{x}^{2} \gamma_{x}\right\| \leq C$ and $\left\|w_{x}\right\|_{C^{0}} \leq C \sqrt{\varepsilon}$ hold for any solution defined on a subinterval $\left[0, T^{\prime}\right)$ of $[0, T)$.

Proof. We consider in a small time interval such that $1 / 2 \leq w \leq 1$ holds by Lemma 2.3. We calculate the time derivative of $\left\|\nabla_{x}^{2} \gamma_{x}\right\|^{2}$ to get

$$
\begin{aligned}
\frac{d}{d t}\left\|\nabla_{x}^{2} \gamma_{x}\right\|^{2} & =2\left\langle\nabla_{x}^{2} \gamma_{x}, \nabla_{t} \nabla_{x}^{2} \gamma_{x}\right\rangle \\
& =2\left\langle\nabla_{x}^{2} \gamma_{x}, R\left(\gamma_{t}, \gamma_{x}\right) \nabla_{x} \gamma_{x}+\nabla_{x}\left(R\left(\gamma_{t}, \gamma_{x}\right) \gamma_{x}\right)+\nabla_{x}^{3} \gamma_{t}\right\rangle
\end{aligned}
$$

The curvature terms are bounded by

$$
C_{3}\left\|\nabla_{x}^{2} \gamma_{x}\right\|\left\|\left|\nabla_{x} \gamma_{x}\right|^{2}+\left|\nabla_{x}^{2} \gamma_{x}\right|\right\| \leq C_{4}\left(1+\left\|\nabla_{x}^{2} \gamma_{x}\right\|^{2}\right) .
$$

For the remaining term $2\left\langle\nabla_{x}^{2} \gamma_{x}, \nabla_{x}^{3} \gamma_{t}\right\rangle$, we have

$$
\begin{aligned}
2\left\langle\nabla_{x}^{2} \gamma_{x}, \nabla_{x}^{3} \gamma_{t}\right\rangle & =-2\left\langle\nabla_{x}^{3} \gamma_{x}, \nabla_{x}^{2} \gamma_{t}\right\rangle \\
& =-2\left\langle\nabla_{x}^{3} \gamma_{x}, \nabla_{x} \gamma_{x} \times \nabla_{x}^{2} \gamma_{x}+\gamma_{x} \times \nabla_{x}^{3} \gamma_{x}+\varepsilon \nabla_{x}^{3} \gamma_{x}\right\rangle \\
& =-2 \varepsilon\left\|\nabla_{x}^{3} \gamma_{x}\right\|^{2}-2\left\langle\nabla_{x}^{3} \gamma_{x}, \nabla_{x} \gamma_{x} \times \nabla_{x}^{2} \gamma_{x}\right\rangle
\end{aligned}
$$

We decompose each factor of $\left\langle\nabla_{x}^{3} \gamma_{x}, \nabla_{x} \gamma_{x} \times \nabla_{x}^{2} \gamma_{x}\right\rangle$ to the $\gamma_{x}$ part and the component perpendicular to $\gamma_{x}$ to get

$$
\begin{aligned}
-2\left\langle\nabla_{x}^{3} \gamma_{x}, \nabla_{x} \gamma_{x} \times \nabla_{x}^{2} \gamma_{x}\right\rangle= & -2\left\langle w^{-1} g\left(\nabla_{x}^{3} \gamma_{x}, \gamma_{x}\right) \gamma_{x}, \nabla_{x} \gamma_{x} \times \nabla_{x}^{2} \gamma_{x}\right\rangle \\
& -2\left\langle\nabla_{x}^{3} \gamma_{x}, w^{-1} g\left(\nabla_{x} \gamma_{x}, \gamma_{x}\right) \gamma_{x} \times \nabla_{x}^{2} \gamma_{x}\right\rangle \\
& -2\left\langle\nabla_{x}^{3} \gamma_{x}, w^{-1} g\left(\nabla_{x}^{2} \gamma_{x}, \gamma_{x}\right) \nabla_{x} \gamma_{x} \times \gamma_{x}\right\rangle .
\end{aligned}
$$


For the first term, we use the equality: $2 g\left(\nabla_{x}^{3} \gamma_{x}, \gamma_{x}\right)=w_{x x x}-3 \partial_{x}\left(\left|\nabla_{x} \gamma_{x}\right|^{2}\right)$. Then

$$
\begin{aligned}
-2\left\langle w^{-1} g\right. & \left.\left(\nabla_{x}^{3} \gamma_{x}, \gamma_{x}\right) \gamma_{x}, \nabla_{x} \gamma_{x} \times \nabla_{x}^{2} \gamma_{x}\right\rangle \\
& =-\left\langle w^{-1}\left\{w_{x x x}-3 \partial_{x}\left(\left|\nabla_{x} \gamma_{x}\right|^{2}\right)\right\} \gamma_{x}, \nabla_{x} \gamma_{x} \times \nabla_{x}^{2} \gamma_{x}\right\rangle \\
& \leq C_{5}\left(\left\|w_{x x x}\right\|+\left\|\nabla_{x} \gamma_{x}\right\|_{C^{0}}\left\|\nabla_{x}^{2} \gamma_{x}\right\|\right)\left\|\nabla_{x} \gamma_{x}\right\|_{C^{0}}\left\|\nabla_{x}^{2} \gamma_{x}\right\| \\
& \leq\left\|w_{x x x}\right\|^{2}+C_{6}\left(1+\left\|\nabla_{x}^{2} \gamma_{x}\right\|^{3}\right) .
\end{aligned}
$$

For the second term, we use the equality: $2 g\left(\nabla_{x} \gamma_{x}, \gamma_{x}\right)=w_{x}$. Then

$$
\begin{aligned}
-2\left\langle\nabla_{x}^{3} \gamma_{x},\right. & \left.w^{-1} g\left(\nabla_{x} \gamma_{x}, \gamma_{x}\right) \gamma_{x} \times \nabla_{x}^{2} \gamma_{x}\right\rangle=-\left\langle\nabla_{x}^{3} \gamma_{x}, w^{-1} w_{x} \gamma_{x} \times \nabla_{x}^{2} \gamma_{x}\right\rangle \\
& \leq C_{7}\left\|w_{x}\right\|_{C^{0}}\left\|\nabla_{x}^{3} \gamma_{x}\right\|\left\|\nabla_{x}^{2} \gamma_{x}\right\| \leq C_{8}\left(\left\|w_{x}\right\|+\left\|w_{x x}\right\|\right)\left\|\nabla_{x}^{3} \gamma_{x}\right\|\left\|\nabla_{x}^{2} \gamma_{x}\right\| \\
& \leq \varepsilon\left\|\nabla_{x}^{3} \gamma_{x}\right\|^{2}+C_{9} \varepsilon^{-1}\left(\left\|w_{x}\right\|^{2}+\left\|w_{x x}\right\|^{2}\right)\left\|\nabla_{x}^{2} \gamma_{x}\right\|^{2} .
\end{aligned}
$$

For the last term, we use the equality: $2 g\left(\nabla_{x}^{2} \gamma_{x}, \gamma_{x}\right)=w_{x x}-2\left|\nabla_{x} \gamma_{x}\right|^{2}$. Then

$$
-2\left\langle\nabla_{x}^{3} \gamma_{x}, w^{-1} g\left(\nabla_{x}^{2} \gamma_{x}, \gamma_{x}\right) \nabla_{x} \gamma_{x} \times \gamma_{x}\right\rangle=2\left\langle\nabla_{x}^{2} \gamma_{x}, \partial_{x}\left\{w^{-1} g\left(\nabla_{x}^{2} \gamma_{x}, \gamma_{x}\right)\right\} \nabla_{x} \gamma_{x} \times \gamma_{x}\right\rangle,
$$

which has bounds similar to the first term.

Summing up these, we have

$$
\begin{aligned}
\frac{d}{d t}\left\|\nabla_{x}^{2} \gamma_{x}\right\|^{2} \leq & -\varepsilon\left\|\nabla_{x}^{3} \gamma_{x}\right\|^{2}+2\left\|w_{x x x}\right\|^{2} \\
& +C_{10}\left\{1+\left\|\nabla_{x}^{2} \gamma_{x}\right\|^{3}+\varepsilon^{-1}\left(\left\|w_{x}\right\|^{2}+\left\|w_{x x}\right\|^{2}\right)\left\|\nabla_{x}^{2} \gamma_{x}\right\|^{2}\right\} .
\end{aligned}
$$

Combining it with Lemma 2.4, we see that $X(t):=\varepsilon^{-1}\left\|w_{x}\right\|^{2}+\varepsilon^{-1}\left\|w_{x x}\right\|^{2}+(1 / 2)\left\|\nabla_{x}^{2} \gamma_{x}\right\|^{2}$ satisfies $X^{\prime}(t) \leq C_{11}(1+X(t))^{2}$. Therefore, $\varepsilon^{-1}\left\|w_{x}\right\|^{2}, \varepsilon^{-1}\left\|w_{x x}\right\|^{2}$ and $\left\|\nabla_{x}^{2} \gamma_{x}\right\|$ are uniformly bounded on a certain finite time interval.

Lemma 2.6. Let $T$ be as in Proposition 2.5 and $n$ a nonnegative integer. For any positive number $K$, there exists a positive constant $C$ such that if $\left\|\gamma_{x}\right\|_{n+2} \leq K$, then

$$
\varepsilon^{-1} \frac{d}{d t}\left\|\partial_{x}^{n+3} w\right\|^{2} \leq-\left\|\partial_{x}^{n+4} w\right\|^{2}+C\left(1+\left\|\nabla_{x}^{n+3} \gamma_{x}\right\|^{2}\right) .
$$

PROOF.

$$
\begin{aligned}
\frac{d}{d t}\left\|\partial_{x}^{n+3} w\right\|^{2} & =2\left\langle\partial_{x}^{n+3} w, \partial_{x}^{n+3} w_{t}=-2 \varepsilon\left\langle\partial_{x}^{n+4} w, \partial_{x}^{n+2}\left(w_{x x}-2\left|\nabla_{x} \gamma_{x}\right|^{2}\right)\right\rangle\right\rangle \\
& \leq-2 \varepsilon\left\|\partial_{x}^{n+4} w\right\|^{2}+4 \varepsilon\left\|\partial_{x}^{n+4} w\right\|\left\|\partial_{x}^{n+2}\left(\left|\nabla_{x} \gamma_{x}\right|^{2}\right)\right\| .
\end{aligned}
$$

Here, we also have

$$
\begin{aligned}
\left\|\partial_{x}^{n+2}\left(\left|\nabla_{x} \gamma_{x}\right|^{2}\right)\right\| & \leq 2\left\|\nabla_{x}^{n+3} \gamma_{x}\right\|\left\|\nabla_{x} \gamma_{x}\right\|_{C^{0}}+C_{1}\left\|\nabla_{x}^{n+2} \gamma_{x}\right\|\left\|\nabla_{x} \gamma_{x}\right\|_{C^{1}}+C_{2} \\
& \leq C_{3}\left(1+\left\|\nabla_{x}^{n+3} \gamma_{x}\right\|\right) .
\end{aligned}
$$


Lemma 2.7. Let $T$ be as in Proposition 2.5 and $n$ a nonnegative integer. For any positive number $K$, there exists a positive constant $C$ such that if $\left\|\gamma_{x}\right\|_{n+2} \leq K$, then

$$
\frac{d}{d t}\left\|\nabla_{x}^{n+3} \gamma_{x}\right\|^{2} \leq-\varepsilon\left\|\nabla_{x}^{n+4} \gamma_{x}\right\|^{2}+C\left(1+\left\|\nabla_{x}^{n+3} \gamma_{x}\right\|^{2}+\left\|\partial_{x}^{n+4} w\right\|^{2}\right) .
$$

PROOF.

$$
\begin{aligned}
\frac{d}{d t} & \left\|\nabla_{x}^{n+3} \gamma_{x}\right\|^{2}=2\left\langle\nabla_{x}^{n+3} \gamma_{x}, \nabla_{t} \nabla_{x}^{n+3} \gamma_{x}\right\rangle \\
& =2\left\langle\nabla_{x}^{n+3} \gamma_{x}, \sum_{i=0}^{n+2} \nabla_{x}^{i}\left(R\left(\gamma_{t}, \gamma_{x}\right) \nabla_{x}^{n+2-i} \gamma_{x}\right)+\nabla_{x}^{n+4} \gamma_{t}\right\rangle \\
& \leq C_{1}\left\|\gamma_{x}\right\|_{n+3}^{2}+2\left\langle\nabla_{x}^{n+3} \gamma_{x}, \nabla_{x}^{n+4}\left(\gamma_{x} \times \nabla_{x} \gamma_{x}+\varepsilon \nabla_{x} \gamma_{x}\right)\right\rangle \\
& =C_{1}\left\|\gamma_{x}\right\|_{n+3}^{2}-2 \varepsilon\left\|\nabla_{x}^{n+4} \gamma_{x}\right\|^{2}+2 \sum_{i=0}^{n+4}\left(\begin{array}{c}
n+4 \\
i
\end{array}\right)\left\langle\nabla_{x}^{n+3} \gamma_{x}, \nabla_{x}^{i} \gamma_{x} \times \nabla_{x}^{n+5-i} \gamma_{x}\right\rangle .
\end{aligned}
$$

In the last summation term, $\left\|\nabla_{x}^{i} \gamma_{x} \times \nabla_{x}^{j} \gamma_{x}\right\| \leq\left\|\nabla_{x}^{i} \gamma_{x}\right\|_{C^{0}}\left\|\nabla_{x}^{j} \gamma_{x}\right\| \leq C_{2}$ if $i<j \leq n+2$, and cancels if $i=2$ or $n+3$. Therefore, we have to measure only terms with $i=0,1, n+4$. Moreover, the term with $i=0$ equals to $-\left\langle\nabla_{x}^{n+3} \gamma_{x}, \nabla_{x} \gamma_{x} \times \nabla_{x}^{n+4} \gamma_{x}\right\rangle$, and is reduced to the case $i=1$.

As in the proof of Proposition 2.5, we decompose each factor of the term with $i=1$ and $n+4$ to the $\gamma_{x}$ part and the component perpendicular to $\gamma_{x}$.

$$
\begin{aligned}
\left\langle\nabla_{x}^{n+3} \gamma_{x}, \nabla_{x} \gamma_{x} \times \nabla_{x}^{n+4} \gamma_{x}\right\rangle= & \left\langle w^{-1} g\left(\nabla_{x}^{n+3} \gamma_{x}, \gamma_{x}\right) \gamma_{x}, \nabla_{x} \gamma_{x} \times \nabla_{x}^{n+4} \gamma_{x}\right\rangle \\
& +\left\langle\nabla_{x}^{n+3} \gamma_{x}, w^{-1} g\left(\nabla_{x} \gamma_{x}, \gamma_{x}\right) \gamma_{x} \times \nabla_{x}^{n+4} \gamma_{x}\right\rangle \\
& +\left\langle\nabla_{x}^{n+3} \gamma_{x}, w^{-1} g\left(\nabla_{x}^{n+4} \gamma_{x}, \gamma_{x}\right) \nabla_{x} \gamma_{x} \times \gamma_{x}\right\rangle .
\end{aligned}
$$

We know that $g\left(\nabla_{x}^{n+3} \gamma_{x}, \gamma_{x}\right)=(1 / 2) \partial_{x}^{n+3} w-C_{3} g\left(\nabla_{x}^{n+2} \gamma_{x}, \nabla_{x} \gamma_{x}\right)+($ lower derivatives). The first term is estimated as

$$
\begin{aligned}
\left\langle w^{-1} g\right. & \left.\left(\nabla_{x}^{n+3} \gamma_{x}, \gamma_{x}\right) \gamma_{x}, \nabla_{x} \gamma_{x} \times \nabla_{x}^{n+4} \gamma_{x}\right\rangle \\
= & -\left\langle\partial_{x}\left\{w^{-1} g\left(\nabla_{x}^{n+3} \gamma_{x}, \gamma_{x}\right)\right\} \gamma_{x}, \nabla_{x} \gamma_{x} \times \nabla_{x}^{n+3} \gamma_{x}\right\rangle \\
& -\left\langle w^{-1} g\left(\nabla_{x}^{n+3} \gamma_{x}, \gamma_{x}\right) \gamma_{x}, \nabla_{x}^{2} \gamma_{x} \times \nabla_{x}^{n+3} \gamma_{x}\right\rangle \\
\leq & C_{4}\left(\left\|\partial_{x}^{n+4} w\right\|+\left\|\gamma_{x}\right\|_{n+3}+\left\|\partial_{x}^{n+3} w\right\|_{C^{0}}+\left\|\gamma_{x}\right\|_{\left.C^{n+2}\right)\left\|\nabla_{x}^{n+3} \gamma_{x}\right\|}\right. \\
\leq & C_{5}\left(1+\left\|\partial_{x}^{n+4} w\right\|+\left\|\nabla_{x}^{n+3} \gamma_{x}\right\|\right)\left\|\nabla_{x}^{n+3} \gamma_{x}\right\| \\
\leq & C_{6}\left(1+\left\|\partial_{x}^{n+4} w\right\|^{2}+\left\|\nabla_{x}^{n+3} \gamma_{x}\right\|^{2}\right) .
\end{aligned}
$$

The last term $\left\langle\nabla_{x}^{n+3} \gamma_{x}, w^{-1} g\left(\nabla_{x}^{n+4} \gamma_{x}, \gamma_{x}\right) \nabla_{x} \gamma_{x} \times \gamma_{x}\right\rangle$ can be estimated similarly. Since $\left|g\left(\nabla_{x} \gamma_{x}, \gamma_{x}\right)\right|=(1 / 2)\left|w_{x}\right| \leq C_{7} \sqrt{\varepsilon}$, the second term is estimated as 


$$
\begin{gathered}
\left\langle\nabla_{x}^{n+3} \gamma_{x}, w^{-1} g\left(\nabla_{x} \gamma_{x}, \gamma_{x}\right) \gamma_{x} \times \nabla_{x}^{n+4} \gamma_{x}\right\rangle \leq C_{8} \sqrt{\varepsilon}\left\|\nabla_{x}^{n+3} \gamma_{x}\right\|\left\|\nabla_{x}^{n+4} \gamma_{x}\right\| \\
\leq a \varepsilon\left\|\nabla_{x}^{n+4} \gamma_{x}\right\|^{2}+C_{9} a^{-1}\left\|\nabla_{x}^{n+3} \gamma_{x}\right\|^{2},
\end{gathered}
$$

where $a$ is an arbitrary positive number.

Summing up these with sufficiently small $a$, we get the result.

THEOREM 2.8. Let $T$ be as in Proposition 2.5. There exists a $C^{\infty}$ solution $\gamma$ of $(\mathrm{VM})$ on $0 \leq t<T$.

Proof. By Lemmas 2.6 and 2.7, $X(t):=\left\|\nabla_{x}^{n+3} \gamma_{x}\right\|^{2}+C \varepsilon^{-1}\left\|\partial_{x}^{n+3} w\right\|^{2}$ satisfies $X^{\prime}(t) \leq C_{1}(1+X(t))$, where $C$ is as in Lemma 2.7. Therefore, by induction, each solution is smoothly bounded. Since the bound is uniform with respect to $t$, we can continue the solution up to $T$. Moreover, since the bounds are independent of $\varepsilon$, a subsequence of $\gamma^{\varepsilon}$ $(\varepsilon \downarrow 0)$ converges smoothly. The limit is a solution of (VM).

3. Uniqueness. Once proving the existence of a solution, to show the uniqueness is standard. We take a tubular neighbourhood $U$ of the initial data $\gamma_{0}$, and embed it in $\boldsymbol{R}^{3}$. In other words, we consider the vortex filament equation in $\boldsymbol{R}^{3}$ with a curved Riemannian metric $g$. With the coordinate of $\boldsymbol{R}^{3}$, we express the covariant differentiation and the exterior product by

$$
\nabla_{x} \alpha=\nabla_{x}\left(\alpha^{i} \partial_{i}\right)=\left(\alpha_{x}^{i}+\Gamma_{j}{ }^{i}{ }_{k} \gamma_{x}^{j} \alpha^{k}\right) \partial_{i}, \quad \alpha \times \beta=\left(\alpha^{j} \partial_{j}\right) \times\left(\beta^{k} \partial_{k}\right)=\chi_{j}{ }^{i} \alpha^{j} \beta^{k} \partial_{i},
$$

where $\partial_{i}$ are the coordinate vector fields, $\Gamma_{j}{ }_{k}{ }_{k}$ are the Christoffel symbols, and $\chi_{j}{ }^{i} k$ are the coordinate expression of the exterior product. Using this, (VM) is written as

$$
\gamma_{t}^{i}=\chi_{j k}^{i} \gamma_{x}^{j}\left(\gamma_{x x}^{k}+\Gamma_{l}^{k}{ }_{m}^{k} \gamma_{x}^{l} \gamma_{x}^{m}\right) .
$$

Let $\eta$ be another solution with the same initial data. By ignoring $\varepsilon$ in Section 2, $\eta$ satisfies the same estimation as $\gamma$. We use $\tilde{\chi}$ and $\tilde{\Gamma}$ the corresponding coefficients along $\eta$, and put $\zeta^{i}:=\eta^{i}-\gamma^{i}$. Then $\zeta^{i}$ satisfies

$$
\zeta_{t}^{i}=\chi_{j}{ }^{i}{ }_{k} \gamma_{x}^{j}\left(\zeta_{x x}^{k}+2 \Gamma_{l}^{k}{ }_{m} \gamma_{x}^{l} \zeta_{x}^{m}\right)+\chi_{j}{ }^{i} \zeta_{x}^{j}\left(\gamma_{x x}^{k}+\Gamma_{l}^{k}{ }_{m} \gamma_{x}^{l} \gamma_{x}^{m}\right)+\chi_{j}{ }^{i} \zeta_{x}^{j} \zeta_{x x}^{k}+P,
$$

where $P$ is a sum of terms that contains $\tilde{\chi}_{j}{ }^{i}{ }_{k}-\chi_{j}{ }^{i}{ }_{k}, \tilde{\Gamma}_{j}{ }^{i}{ }_{k}-\Gamma_{j}{ }^{i}{ }_{k}$ or $\zeta_{x}{ }^{i} \zeta_{x}^{j}$. Since $\gamma$ and $\zeta=\eta-\gamma$ are smoothly bounded, we know that $|P|,\left|P_{x}\right| \leq C_{1}\left(|\zeta|+\left|\zeta_{x}\right|\right)$.

We identify $\zeta$ with a vector field $\zeta^{i} \partial_{i}$ along $\gamma$. Then we obtain

$$
\begin{aligned}
& \nabla_{t} \zeta=\left(\zeta_{t}^{i}+\Gamma_{j}{ }^{i}{ }_{k} \gamma_{t}^{j} \zeta^{k}\right) \partial_{i}, \\
& \nabla_{x} \zeta=\left(\zeta_{x}^{i}+\Gamma_{j}{ }_{k}{ }_{k} \gamma_{x}^{j} \zeta^{k}\right) \partial_{i}, \\
& \nabla_{x}^{2} \zeta=\left(\zeta_{x x}^{i}+2 \Gamma_{j}{ }_{k}{ }_{k} \gamma_{x}^{j} \zeta_{x}^{k}+\left(\Gamma_{j}{ }_{k}{ }_{k} \gamma_{x}^{j}\right)_{x} \zeta^{k}+\Gamma_{j}{ }_{k}{ }_{k} \gamma_{x}^{j} \Gamma_{l}{ }_{m}{ }_{m} \gamma_{x}^{l} \zeta^{m}\right) \partial_{i} .
\end{aligned}
$$

Substituting these to (3.1), we get

$$
\nabla_{t} \zeta=\gamma_{x} \times \nabla_{x}^{2} \zeta+\nabla_{x} \zeta \times \nabla_{x} \gamma_{x}+\nabla_{x} \zeta \times \nabla_{x}^{2} \zeta+Q,
$$

where $Q$ is a sum of $P$ and terms that contain $\zeta^{i}$ or $\zeta_{x}^{i} \zeta_{x}^{j}$. Note that $|Q|,\left|\nabla_{x} Q\right| \leq$ $C_{2}\left(|\zeta|+\left|\nabla_{x} \zeta\right|\right)$. 
Therefore, we have

$$
\begin{aligned}
\frac{d}{d t}\|\zeta\|^{2} & =2\left\langle\zeta, \nabla_{t} \zeta\right\rangle=2\left\langle\zeta, \gamma_{x} \times \nabla_{x}^{2} \zeta+\nabla_{x} \zeta \times \nabla_{x} \gamma_{x}+\nabla_{x} \zeta \times \nabla_{x}^{2} \zeta+Q\right\rangle \\
& =-2\left\langle\zeta, \nabla_{x} \gamma_{x} \times \nabla_{x} \zeta\right\rangle+2\left\langle\zeta, \nabla_{x} \zeta \times \nabla_{x} \gamma_{x}\right\rangle+2\left\langle\zeta, \nabla_{x} \zeta \times \nabla_{x}^{2} \zeta\right\rangle+2\langle\zeta, Q\rangle \\
& \leq C_{3}\|\zeta\|\left(\|\zeta\|+\left\|\nabla_{x} \zeta\right\|\right),
\end{aligned}
$$

because $\nabla_{x}^{2} \zeta$ is bounded. Also, we have

$$
\begin{aligned}
\frac{d}{d t}\left\|\nabla_{x} \zeta\right\|^{2} & =2\left\langle\nabla_{x} \zeta, \nabla_{t} \nabla_{x} \zeta\right\rangle=2\left\langle\nabla_{x} \zeta, R\left(\gamma_{t}, \gamma_{x}\right) \zeta+\nabla_{x} \nabla_{t} \zeta\right\rangle \\
& \leq C_{4}\|\zeta\|\left\|\nabla_{x} \zeta\right\|-2\left\langle\nabla_{x}^{2} \zeta, \nabla_{t} \zeta\right\rangle \\
& =C_{4}\|\zeta\|\left\|\nabla_{x} \zeta\right\|-2\left\langle\nabla_{x}^{2} \zeta, \nabla_{x} \zeta \times \nabla_{x} \gamma_{x}+Q\right\rangle \\
& =C_{4}\|\zeta\|\left\|\nabla_{x} \zeta\right\|-2\left\langle\nabla_{x}^{2} \zeta, \nabla_{x} \zeta \times \nabla_{x} \gamma_{x}\right\rangle+2\left\langle\nabla_{x} \zeta, \nabla_{x} Q\right\rangle \\
& \leq C_{5}\|\zeta\|\left(\|\zeta\|+\left\|\nabla_{x} \zeta\right\|\right)-2\left\langle\nabla_{x}^{2} \zeta, \nabla_{x} \zeta \times \nabla_{x} \gamma_{x}\right\rangle .
\end{aligned}
$$

To estimate the remaining term, we use the equality $g\left(\gamma_{x}, \gamma_{x}\right)=1$. By the same way as the case of $\Gamma$ and $\chi$, it implies that $g\left(\gamma_{x}, \nabla_{x} \zeta\right)$ can be expressed as a sum of terms that contain $\tilde{g}_{i j}-g_{i j}, \zeta^{i}$ or $\zeta_{x}^{i} \zeta_{x}^{j}$, and we have $\left|g\left(\gamma_{x}, \nabla_{x} \zeta\right)\right|,\left|\partial_{x}\left(g\left(\gamma_{x}, \nabla_{x} \zeta\right)\right)\right|,\left|g\left(\gamma_{x}, \nabla_{x}^{2} \zeta\right)\right| \leq$ $C_{6}\left(|\zeta|+\left|\nabla_{x} \zeta\right|\right)$. Since $\nabla_{x} \gamma_{x}$ is perpendicular to $\gamma_{x}$,

$$
\begin{aligned}
\left\langle\nabla_{x}^{2} \zeta, \nabla_{x} \zeta \times \nabla_{x} \gamma_{x}\right\rangle & =\left\langle g\left(\nabla_{x}^{2} \zeta, \gamma_{x}\right) \gamma_{x}, \nabla_{x} \zeta \times \nabla_{x} \gamma_{x}\right\rangle+\left\langle\nabla_{x}^{2} \zeta, g\left(\nabla_{x} \zeta, \gamma_{x}\right) \gamma_{x} \times \nabla_{x} \gamma_{x}\right\rangle \\
& =\left\langle g\left(\nabla_{x}^{2} \zeta, \gamma_{x}\right) \gamma_{x}, \nabla_{x} \zeta \times \nabla_{x} \gamma_{x}\right\rangle-\left\langle\nabla_{x} \zeta, \nabla_{x}\left\{g\left(\nabla_{x} \zeta, \gamma_{x}\right) \gamma_{x} \times \nabla_{x} \gamma_{x}\right\}\right\rangle \\
& \leq C_{7}\left\|\nabla_{x} \zeta\right\|\left(\|\zeta\|+\left\|\nabla_{x} \zeta\right\|\right) .
\end{aligned}
$$

Therefore, $X(t):=\|\zeta\|^{2}+\left\|\nabla_{x} \zeta\right\|^{2}$ satisfies $X^{\prime}(t) \leq C_{8} X(t)$, which implies that $\zeta$ identically vanishes. We have proved

THEOREM 3.1. (VM) has a unique short time solution for any closed initial curve $\gamma_{0}(x)$ with $\left|\gamma_{0 x}\right| \equiv 1$.

4. Appendix. In Section 2, we heavily used the fact that the time derivative of $w$ is bounded by $\varepsilon$. There is another method of estimation, which we did not use because it is lengthier than the proof given in Section 2. The method uses a weighted norm that has resemblance to $[\mathrm{N}]$. Therefore, there may be some interest to the method. Here, we give its key point.

Since the $\varepsilon$-parts are easy to estimate by usual parabolic equation's argument, we can ignore such terms. Also, we can ignore curvature terms, because they contain only lower derivatives.

Let $\varphi$ be the part of $\nabla_{x}^{2} \gamma_{x}$ perpendicular to $\gamma_{x}$. Namely,

$$
\varphi:=\nabla_{x}^{2} \gamma_{x}-u \gamma_{x} ; \quad u:=w^{-1} g\left(\nabla_{x}^{2} \gamma_{x}, \gamma_{x}\right)=w^{-1}\left\{\left(w_{x x} / 2\right)-\left|\nabla_{x} \gamma_{x}\right|^{2}\right\} .
$$


By Lemmas 2.2 and 2.3, we know that $\left\|\nabla_{x} \gamma_{x}\right\|$ and $\left\|w_{x}\right\|$ are bounded from above, and that $w$ is bounded from below. Therefore,

$$
\begin{aligned}
& \partial_{x}\left|\nabla_{x} \gamma_{x}\right|^{2}=2 g\left(\nabla_{x} \gamma_{x}, \nabla_{x}^{2} \gamma_{x}\right)=2 g\left(\nabla_{x} \gamma_{x}, \varphi+u \gamma_{x}\right), \\
&\left\|\partial_{x}\left|\nabla_{x} \gamma_{x}\right|\right\| \leq \leq \varphi \varphi+\|u\| \leq C_{1}\left(\|\varphi\|+\left\|w_{x x}\right\|+\left\|\nabla_{x} \gamma_{x}\right\|_{C^{0}}\right) \\
& \leq \frac{1}{2}\left\|\partial_{x}\left|\nabla_{x} \gamma_{x}\right|\right\|+C_{2}\left(\|\varphi\|+\left\|w_{x x}\right\|+1\right), \\
&\left\|\nabla_{x} \gamma_{x}\right\|_{C^{0}} \leq C_{3}\left(\|\varphi\|+\left\|w_{x x}\right\|+1\right), \\
&\left\|\nabla_{x}^{2} \gamma_{x}\right\|^{2}=\|\varphi\|^{2}+\left\|u \gamma_{x}\right\|^{2} \leq C_{4}\left(\|\varphi\|^{2}+\left\|w_{x x}\right\|^{2}+\left\|\nabla_{x} \gamma_{x}\right\|_{C^{0}}\right) \\
& \leq C_{5}\left(\|\varphi\|^{2}+\left\|w_{x x}\right\|^{2}+1\right),
\end{aligned}
$$

which imply that we can use $\|\varphi\|$ instead of $\left\|\nabla_{x}^{2} \gamma_{x}\right\|$. Note also that $w_{x x}$ contains third derivatives of $\gamma$, and is comparable to $\varphi$. From

$$
\begin{aligned}
& w_{t}=[\varepsilon \text { terms }], \\
& \gamma_{t}=\gamma_{x} \times \nabla_{x} \gamma_{x}+\varepsilon \nabla_{x} \gamma_{x}=\gamma_{x} \times \nabla_{x} \gamma_{x}+[\varepsilon \text { terms }], \\
& \nabla_{x} \gamma_{t}=\gamma_{x} \times \nabla_{x}^{2} \gamma_{x}+[\varepsilon \text { terms }]=\gamma_{x} \times \varphi+[\varepsilon \text { terms }],
\end{aligned}
$$

we have

$$
\begin{aligned}
\nabla_{t} \varphi & =\nabla_{t} \nabla_{x}^{2} \gamma_{x}-u_{t} \gamma_{x}-u \nabla_{x} \gamma_{t} \\
& =\nabla_{x}^{2}\left(\gamma_{x} \times \varphi\right)-u_{t} \gamma_{x}-u \gamma_{x} \times \varphi+[\varepsilon, \text { lower terms }] .
\end{aligned}
$$

For a constant $a$, we put $X(t):=\left\|w^{a} \varphi\right\|^{2}+\left\|w_{x x}\right\|^{2}$. Then we have

$$
\begin{aligned}
\frac{d}{d t}\left\|w^{a} \varphi\right\|^{2} & =2\left\langle w^{2 a} \varphi, \nabla_{t} \varphi\right\rangle+[\varepsilon \text { terms }]=2\left\langle w^{2 a} \varphi, \nabla_{x}^{2}\left(\gamma_{x} \times \varphi\right)\right\rangle+[\varepsilon, \text { lower terms }] \\
& =-2\left\langle\partial_{x}\left(w^{2 a}\right) \varphi+w^{2 a} \nabla_{x} \varphi, \nabla_{x} \gamma_{x} \times \varphi+\gamma_{x} \times \nabla_{x} \varphi\right\rangle+[\varepsilon, \text { lower terms }] \\
& =-4 a\left\langle w^{2 a-1} w_{x} \varphi, \gamma_{x} \times \nabla_{x} \varphi\right\rangle-2\left\langle w^{2 a} \nabla_{x} \varphi, \nabla_{x} \gamma_{x} \times \varphi\right\rangle+[\varepsilon, \text { lower terms }]
\end{aligned}
$$

Here,

$$
\begin{aligned}
-2 & \left\langle w^{2 a} \nabla_{x} \varphi, \nabla_{x} \gamma_{x} \times \varphi\right\rangle \\
& =-2\left\langle w^{2 a-1} g\left(\nabla_{x} \varphi, \gamma_{x}\right) \gamma_{x}, \nabla_{x} \gamma_{x} \times \varphi\right\rangle-2\left\langle w^{2 a-1} \nabla_{x} \varphi, g\left(\nabla_{x} \gamma_{x}, \gamma_{x}\right) \gamma_{x} \times \varphi\right\rangle \\
& =2\left\langle w^{2 a-1} g\left(\varphi, \nabla_{x} \gamma_{x}\right) \gamma_{x}, \nabla_{x} \gamma_{x} \times \varphi\right\rangle-\left\langle w^{2 a-1} w_{x} \nabla_{x} \varphi, \gamma_{x} \times \varphi\right\rangle .
\end{aligned}
$$

Therefore,

$$
\begin{aligned}
\frac{d}{d t}\left\|w^{a} \varphi\right\|^{2} & =(4 a-1)\left\langle w^{2 a-1} w_{x} \nabla_{x} \varphi, \gamma_{x} \times \varphi\right\rangle+2\|\varphi\|^{2}\left\|\nabla_{x} \gamma_{x}\right\|_{C^{0}}^{2}+[\varepsilon, \text { lower terms }] \\
& \leq(4 a-1)\left\langle w^{2 a-1} w_{x} \nabla_{x} \varphi, \gamma_{x} \times \varphi\right\rangle+C_{6}\left(1+X(t)^{2}\right)+[\varepsilon \text { terms }] .
\end{aligned}
$$

For $a=1 / 4$, we have $X^{\prime}(t) \leq C_{7}\left(1+X(t)^{2}\right)$, and $X(t)$ is bounded on a certain finite time interval $[0, T)$. For higher derivatives, we can check by a similar calculation that $X_{n}(t):=\left\|w^{(n+1) / 4} \nabla_{x}^{n} \varphi\right\|^{2}+\left\|\partial_{x}^{n+2} w\right\|^{2}$ satisfies $X_{n}^{\prime}(t) \leq C_{8}\left(1+X_{n}(t)\right)$, where $C_{8}$ depends on $X_{n-1}$. Thus, by induction, we can estimate all derivatives on $[0, T)$. 


\section{REFERENCES}

[E] D. E. Eidelman, Parabolic systems, North-Holland publ., Amsterdam, 1969.

[H] H. HAsimoto, A soliton on a vortex filament, J. Fluid Mech. 51 (1972), 477-485.

[K] N. KoIso, The vortex filament equation and a semilinear Schrödinger equation in a hermitian symmetric space, Osaka J. Math. 34 (1997), 199-214.

[N] T. NishiYAmA, Existence of a solution to the mixed problem for a vector filament equation with an external flow term, J. Math. Sci. Univ. Tokyo 7 (2000), 35-55.

[NT] T. NishiYAma AND A. TANI, Initial and initial-boundary value problems for a vortex filament with or without axial flow, SIAM J. Math. Anal. 27 (1996), 1015-1023.

DEPARTMENT OF MATHEMATICS

FACULTY OF SCIENCE

OSAKA UNIVERSITY

TOYONAKA, OSAKA, 560-0043

JAPAN 